\title{
Anti-arthritic activity of D-carvone against complete Freund's adjuvant-induced arthritis in rats through modulation of inflammatory cytokines
}

\author{
Guifang Chen', Yuxiu Song ${ }^{2}$, Fang $\mathrm{Ma}^{3}$, and Yuxia $\mathrm{Ma}^{3, *}$ \\ ${ }^{1}$ Rheumatism and Immunology Ward of Integrated Traditional Chinese and Western Medicine, ${ }^{2}$ Department of Dermatology Pain Management, ${ }^{3}$ Department \\ of Rheumatology and Immunology, Central Hospital Affiliated to Shandong First Medical University, Jinan, Shandong Province 250013, P. R. China
}

\section{ARTICLE INFO}

Received January 10, 2020

Revised May 20, 2020

Accepted August 11, 2020

\section{*Correspondence}

Yuxia Ma

E-mail: mayuxiajn@sina.com

Key Words

Arthritis

D-carvone

Inflammatory cytokines

Oxidative stress

\begin{abstract}
Chronic joint pain due to loss of cartilage function, degradation of subchondral bone, and related conditions are common plights of an arthritis patient. Antioxidant compounds could solve the problems in arthritic condition. The objective of this study was to evaluate the anti-arthritic activity of D-carvone against complete Freund's adjuvant (CFA)-induced arthritis in rats. D-carvone was orally administered for 25 days at the doses of 30 and $60 \mathrm{mg} / \mathrm{kg}$ against CFA-induced arthritic rats. Changes in body weight, paw swelling, organ index, hematological parameters, oxidative stress markers, inflammatory cytokines, and histopathology were recorded. Oral treatment of D-carvone significantly improved the body weight, reduced the paw swelling, edema formation, and organ index in arthritic rats. The levels of white blood cells were reduced, red blood cells and hemoglobin levels were improved in D-carvone treated arthritic rats. Lipid peroxidation levels were lowered whereas enzymatic and non-enzymatic antioxidants were significantly elevated by D-carvone administration against arthritic rats. D-carvone significantly modulated inflammatory cytokine levels and improved the ankle joint pathology against CFA-induced arthritic inflammation. In conclusion, D-carvone proved significant anti-arthritic activity against CFA-induced arthritis in rats.
\end{abstract}

\section{INTRODUCTION}

Osteoarthritis is a common arthritic condition that causes chronic joint pain for affected individuals. Osteophyte formations, cartilage degradation, changes in subchondral bone, and loss of cartilage function are the common characteristics of osteoarthritis [1]. Cartilage degeneration is a non-reversible process, which was initially attributed to ageing, and genetic factors but recent studies have found that inflammatory reaction causes the condition [2,3]. Mediators of inflammation such as cytokines, chemokines, and reactive oxygen species (ROS) produced in the joint tissues triggers the degradation of cartilage. Pro-inflammatory cytokines such as interleukin (IL)-6, IL-1 $\beta$, and tumor necrosis factor (TNF)- $\alpha$ are directly involved in the disruption of homeostasis in the articular joints and cartilage matrix while inducing the chondrocytes to produce more inflammatory cytokines and nitric oxide, which further amplifies an injurious cellular response [4]. On the other hand, oxidative stress due to overproduction of ROS is contributes to worsen the pathological condition of osteoarthritis by damaging the cartilage matrix [5]. Matrix metalloproteinases released during the inflammatory reaction and oxidative stress conditions also degrades the cartilage, leading to deliberate chronic pain in osteoarthritic patients $[6,7]$. There are measures to overcome the pain in osteoarthritic patients but the need to prevent cartilage degeneration is preferred as a potential solution for osteoarthritis. Previous reports have

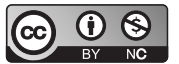

This is an Open Access article distributed under the terms of the Creative Commons Attribution Non-Commercial License, which permits unrestricted non-commercial use, distribution, and reproduction in any medium, provided the original work is properly cited. Copyright @ Korean J Physiol Pharmacol, pISSN 1226-4512, elSSN 2093-3827
Author contributions: G.C., Y.S., F.M., and Y.M. contributed equally in manuscript preparation, experimental data collections, and analysis. 
suggested attenuation of inflammation and oxidative stress could be beneficial in prevention of damage to articular cartilage [8-10].

Plants are natural sources of antioxidants that are acclaimed for numerous medicinal and pharmacological values including antiinflammatory and oxidative stress preventing effects $[9,10]$. Given the involvement of oxidative stress and inflammatory response in the pathogenesis of osteoarthritis, importance was specified to plant-derived antioxidant compounds as the potential solution. D-carvone is an isomer of the monoterpene compound carvone, found in several aromatic plants, which has been reported to possess therapeutic effects such as immunomodulatory, anti-inflammatory, anti-nociceptive, anti-tumorigenic, anti-carcinogenic, anti-hyperlipidemic, anti-hypertensive, and chemopreventive on animal models and cell cultures [11-14]. To our knowledge, there are no reports on the anti-arthritic activity of D-carvone on animal or human model of osteoarthritis till date. Therefore, this research was aimed to evaluate the anti-arthritic activity of D-carvone against complete Freund's adjuvant (CFA)-induced arthritis in rat models.

\section{METHODS}

\section{Chemicals}

D-carvone, indomethacin and CFA were purchased from z, St. Louis, MO, USA. Biochemical assay reagents, diagnostic kits, and ELISA assay kits for cytokines were purchased from Aviva Systems Biology, Beijing, China. All chemicals used were analytical grade in purest quality.

\section{Animals and experimental design}

Wistar albino rats (150-200 g) were randomly divided into five groups, $(n=8)$ in each group. The animals were placed in controlled temperature, ventilation, humidity, and allowed free access to food and water ad libitum. The experimental protocol was approved by the ethics committee of Jinan Central Hospital Affiliated to Shandong University following the stipulated guidelines of the animal welfare and ethics (ethical no.: JXC20180820). Briefly, animals of group I served as normal control (orally administered with saline for the whole experimental period), animals of group II served as arthritic control (given an intradermal injection with $0.1 \mathrm{ml}$ of CFA on the right hind limb on day 1), animals of group III and IV served as experimental groups (given an intradermal injection with $0.1 \mathrm{ml}$ of CFA on the right hind limb on day 1 , followed by oral administration with 30 and $60 \mathrm{mg} / \mathrm{kg}$ of D-carvone once a day for 25 consecutive days), and animals of group $\mathrm{V}$ served as positive control (given an intradermal injection with $0.1 \mathrm{ml}$ of CFA on the right hind limb on day 1 , followed by single oral administration with $3 \mathrm{mg} / \mathrm{kg}$ of indomethacin twice in a week until end of experiment). On the 26 th day after $24 \mathrm{~h}$ of last dosage administration of drugs, the rats were anaesthetized with isoflurane to collect blood samples and sacrificed through cervical dislocation to obtain ankle joint tissues for biochemical and histopathological analysis. The dosages were selected based on the study of Chen et al. [15]. Spleen and thymus of the animals were excised, cleaned and weighed to calculate the organ index (ratio of wet organ to final body weight).

\section{Measurement of paw volume and arthritic score}

The hind paw volume of animals was measured from the initiation of experiment before injection with CFA up to the end of experiment, using a Plethysmometer (UGO Basile 37140; Comerio, Varese, Italy). The measurement of paw volume and visual arthritic scoring were performed by an observer who was not aware of the experimental groups. Visual arthritic scoring was performed by the observer beginning from day 0 until end of experiment, at every 5 day interval. The scoring was given according to the observation of swelling, erythema, and redness in both the hind paws of rats, and separate scores were given for each paw. The scoring was ranged from 0 (normal paw with no swelling or erythema) -4 (severe deformity of the paw with swelling and erythema), and a maximum combined score of 8 was fixed for both hind paws of each rat.

\section{Analysis of serum hematology and biochemistry}

Blood serum collected from the animals were subjected to hematological parameters of white blood cell count (WBC), red blood cell count (RBC), hemoglobin count (Hb), along with serum biochemistry of alkaline phosphatase (ALP), alanine aminotransferase (ALT), and aspartate aminotransferase (AST) levels. All tests were performed using automated laboratory hematological analyzer following standard protocols. Serum malondialdehyde (MDA), reduced glutathione (GSH), and antioxidant enzymes; superoxide dismutase (SOD), and catalase (CAT) levels were measured according to the protocols of standard diagnostic kits (Sigma diagnostic kits; Sigma Aldrich) [15].

\section{ELISA assay for inflammatory cytokine analysis}

Serum biochemical analysis of inflammatory cytokines IL-6, IL-1 $\beta$, IL-10, and TNF- $\alpha$ were performed using standard ELISA assay diagnostic kits (R\&D Systems, Minneapolis, MN, USA) following the manufacturer's instructions. The measurements were standardized for each sample in all groups.

\section{Histopathological examination}

Hind paw tissues of rats fixed in $10 \%$ buffered formalin were trimmed and decalcified in 5\% formic acid. The samples were processed, fixed in paraffin, and sectioned to $5 \mu \mathrm{m}$ for hematoxy- 
lin and eosin (H\&E) staining. The stained sections were studied under light microscope at $100 \times$ magnification for changes due to CFA and drug administration. The histopathological injuries were scored using Mankin's scoring system with some modifications as explained by Jeong et al. [5]. The total Mankin's scoring was given for the articular injuries and cellular irregularities upon histopathological observations by an observer who was not aware of the experimental groupings. The scores ranged from 0-6 for articular injuries with 0 (no visible injury or normal) to 6 (heavily disorganized structure, clusters, calcified cartilage layer), whereas $0-3$ for cellular irregularities with 0 (normal) to 3 (hypocellularity).

\section{Statistical analysis}

All the values were given as mean \pm standard error of mean for eight animals $(n=8)$. The statistical significance for each experiment was evaluated using one-way analysis of variance (ANOVA) and Tukey's multiple comparisons test, SPSS software version 21.0 (IBM Co., Armonk, NY, USA). Values of $\mathrm{p}<0.05$ were regarded significant.

\section{RESULTS}

\section{D-carvone improves body weight, paw volume, arthritic score, and organ index of CFA-induced arthritic rats}

Animals of normal control group is also known as group I, animals of CFA-induced arthritis model group are also known as group II, animals of D-carvone administered experimental groups are also known as group III and IV, whereas animals of indomethacin treated positive control group is also known as group V. Body weight of rats in arthritis model group II was lower $(\mathrm{p}<0.05)$ compared to normal control rats of group I. D-carvone treated rats showed significant improvement on the body weights. Rats in arthritic control group showed significant increase $(\mathrm{p}<$ 0.05 ) in the thymus and spleen index compared to normal control rats. Thymus and spleen index were reduced by D-carvone administration. The results are given in Table 1.

The rats of arthritic model group showed significant paw swelling, redness and erythema in contrast to normal paw of the rats in group I. Paw volumes of rats induced with CFA were increasing for all groups except for normal control group I rats until the first fourteen days. Oral administration of D-carvone remarkably reduced $(\mathrm{p}<0.05)$ the paw volume from the tenth day onwards but the arthritis model rats in group II exhibited increased paw volume until end of experiment. Positive control rats in group $\mathrm{V}$ showed significant amelioration against CFA-induced changes. The results are shown in Fig. 1. The arthritic score given for arthritis model rats in group II were significantly high $(\mathrm{p}<0.05)$ as compared to normal rats in group I. D-carvone treated rats in group III and IV showed significantly lower $(\mathrm{p}<0.05)$ arthritic scores as compared to rats in model group II. The results are displayed in Fig. 2.

\section{Effects of D-carvone on CFA-induced hematological changes in rats}

Arthritis model group rats exhibited increased WBC $(\mathrm{p}<0.05)$ and reduced $\mathrm{RBC}$ and $\mathrm{Hb}(\mathrm{p}<0.05)$ as compared to the normal control rats. Rats administered with D-carvone showed significant increase of RBC, $\mathrm{Hb}(\mathrm{p}<0.05)$ and a decrease in WBC $(\mathrm{p}<$ 0.05 ) in contrast to the arthritic model rats. Positive control group rats also exhibited significant increase in $\mathrm{RBC}, \mathrm{Hb}(\mathrm{p}<0.05)$ and reduced WBC $(\mathrm{p}<0.05)$ as compared to arthritis model group rats. The results are given in Table 2.

\section{D-carvone attenuates serum biochemical changes in CFA-induced arthritic rats}

The serum levels of liver damage marker enzymes ALP, AST, and ALT were significantly elevated $(p<0.05)$ in arthritis model rats of group II compared to normal rats of group I. As shown in Fig. 3 , the rats administered with D-carvone at low and high doses remarkably reduced $(\mathrm{p}<0.05)$ the levels of serum ALP, AST,

Table 1. Changes in body weight, spleen and thymus index of CFA-induced arthritic rats

\begin{tabular}{lcccc}
\hline Groups & Initial body weight $(\mathrm{g})$ & Final body weight $(\mathrm{g})$ & Thymus index $(\mathrm{mg} / \mathrm{g})$ & Spleen index $(\mathrm{mg} / \mathrm{g})$ \\
\hline Group I & $168.2 \pm 10.3$ & $198.5 \pm 7.2$ & $2.24 \pm 0.10$ & $1.42 \pm 0.08$ \\
Group II & $161.6 \pm 8.2$ & $148.4 \pm 13.1^{\mathrm{a}}$ & $2.86 \pm 0.13^{\mathrm{a}}$ & $2.88 \pm 0.11^{\mathrm{a}}$ \\
Group III & $165.3 \pm 9.8$ & $176.7 \pm 8.4^{\mathrm{b}}$ & $2.63 \pm 0.09^{\mathrm{b}}$ & $2.42 \pm 0.09^{\mathrm{b}}$ \\
Group IV & $167.6 \pm 9.3$ & $188.6 \pm 7.6^{\mathrm{b}, \mathrm{c}}$ & $2.41 \pm 0.08^{\mathrm{b}, \mathrm{c}}$ & $1.93 \pm 0.10^{\mathrm{b}, \mathrm{c}}$ \\
Group V & $170.5 \pm 8.6$ & $193.8 \pm 6.8^{\mathrm{b}, \mathrm{c}}$ & $2.29 \pm 0.11^{\mathrm{b}, \mathrm{c}}$ & $1.65 \pm 0.09^{\mathrm{b}, \mathrm{c}}$ \\
\hline
\end{tabular}

Values expressed as mean \pm standard error of mean $(n=8)$ of final body weight and organ index of CFA-induced rats and D-carvone administered rats. Group I = Normal control; Group II = CFA-induced arthritis model; Group III = D-carvone 30 mg/kg b.w. + CFA; Group IV = D-carvone $60 \mathrm{mg} / \mathrm{kg}$ b.w. + CFA; Group V = Indomethacin positive control + CFA. CFA, complete Freund's adjuvant; b.w., body weight. Superscript ' $a$ ' indicates significant difference $(p<0.05)$ from Group I, superscript 'b' indicates significant difference $(p<$ $0.05)$ from Group II, superscript ' $c$ ' indicates significant difference $(p<0.05)$ from Group III, by one-way ANOVA followed by Tukey's multiple comparisons test. 


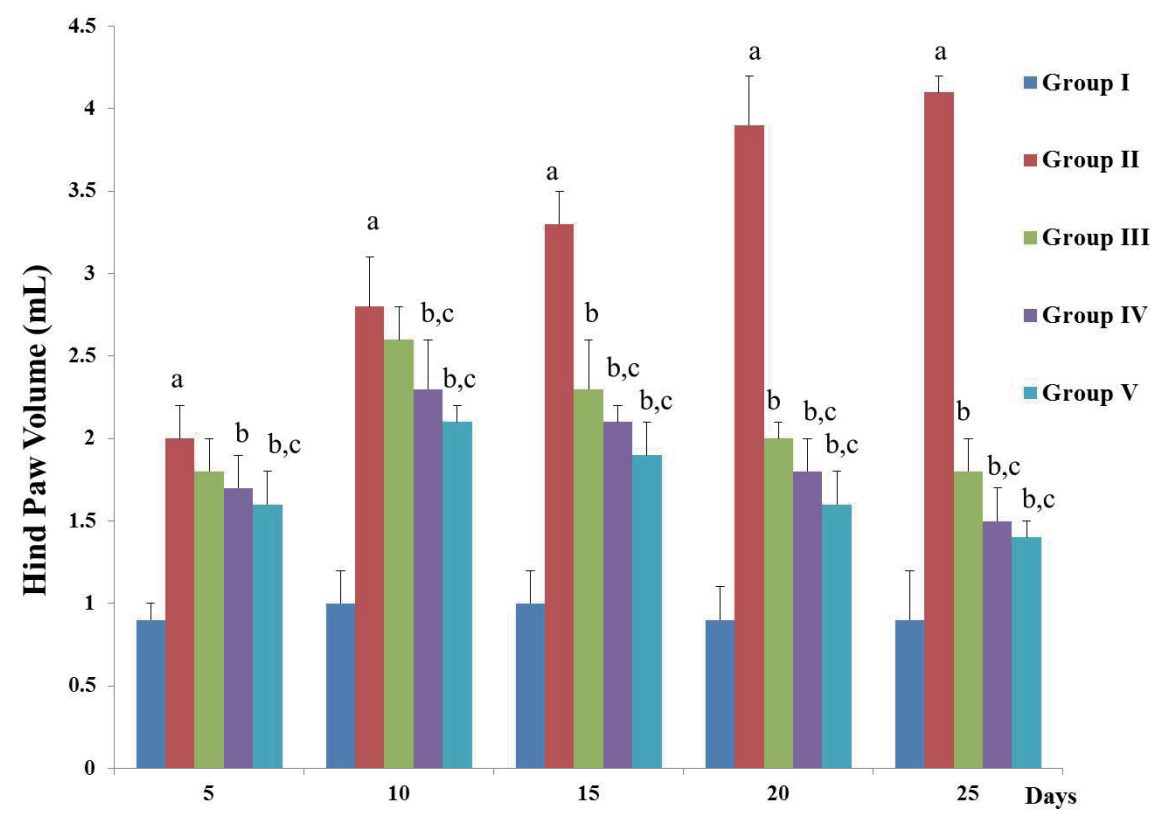

Fig. 1. D-carvone reduces the hind paw volume of CFA-induced arthritic rats. Values expressed as mean \pm standard error of mean of $(n=8)$ rats. Group I = Normal control; Group II = CFA-induced arthritis model; Group III = D-carvone 30 mg/kg b.w. + CFA; Group IV = D-carvone 60 mg/kg b.w. + CFA; Group V = Indomethacin positive control + CFA. CFA, complete Freund's adjuvant; b.w., body weight. Superscript 'a' indicates significant difference $(p<0.05)$ from Group I, superscript ' $b$ ' indicates significant difference $(p<0.05)$ from Group II, superscript ' $c$ ' indicates significant difference $(p<$ 0.05 ) from Group III, by one-way ANOVA followed by Tukey's multiple comparisons test.

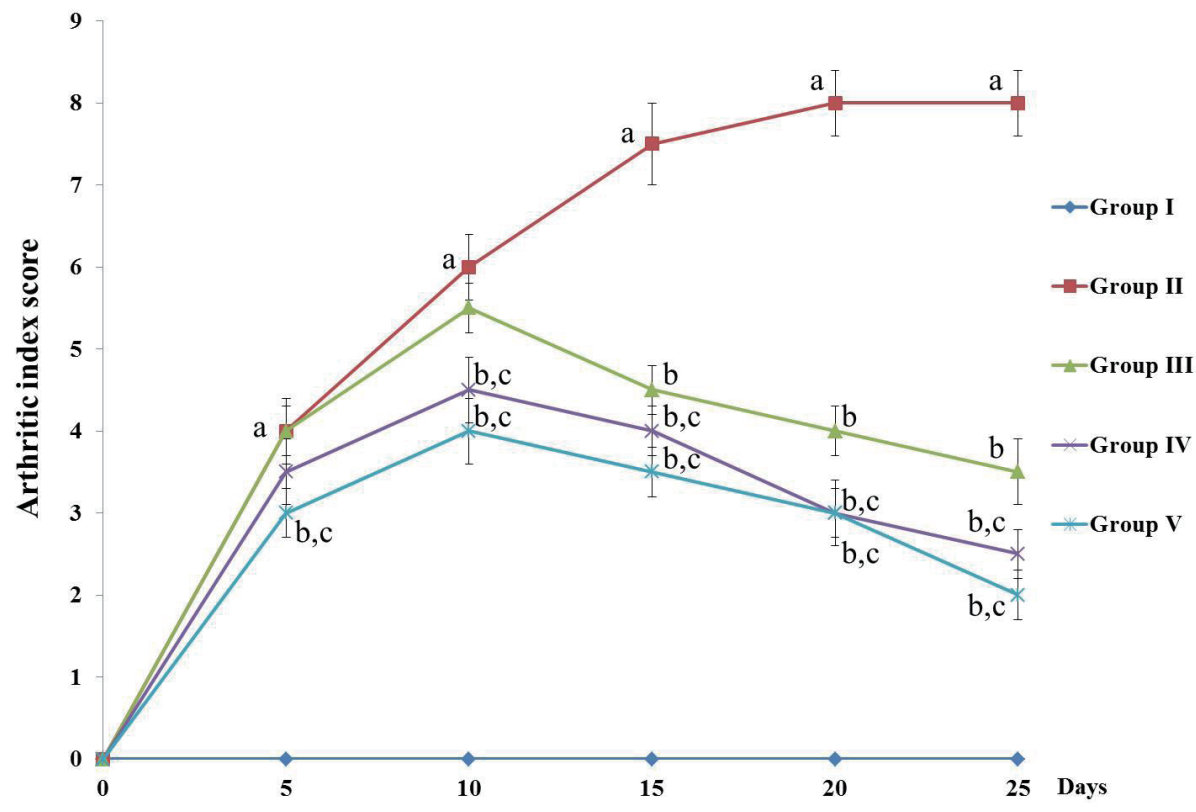

Fig. 2. D-carvone alters the arthritic score of CFA-induced inflammation in rats. Values expressed as mean \pm standard error of mean of $(n=8)$ rats. The arthritic score was ranged from 0 (normal paw with no swelling or erythema) 4 (severe deformity of the paw with swelling and erythema) for a maximum score of 8 for both hind paws. Group I = Normal control; Group II = CFA-induced arthritis model; Group III = D-carvone $30 \mathrm{mg} / \mathrm{kg}$ b.w. + CFA; Group IV = D-carvone 60 mg/kg b.w. + CFA; Group V = Indomethacin positive control + CFA. CFA, complete Freund's adjuvant; b.w., body weight. Superscript ' $a$ ' indicates significant difference $(p<0.05)$ from Group I, superscript ' $b$ ' indicates significant difference $(p<0.05)$ from Group II, superscript ' $c$ ' indicates significant difference $(p<0.05)$ from Group III, by one-way ANOVA followed by Tukey's multiple comparisons test.

and ALT compared to arthritis model rats. Positive control rats of group V showed significantly reduced $(\mathrm{p}<0.05)$ levels of serum ALP, AST, and ALT compared to arthritic model rats of group II.
The levels of MDA formation were increased $(\mathrm{p}<0.05)$ in arthritis model rats of group II compared to normal rats of group I. Oral administration of D-carvone significantly reduced $(\mathrm{p}<$ 
Table 2. Effects of D-carvone on hematological changes in CFA-induced arthritic rats

\begin{tabular}{lccc}
\hline Group & WBC $\left(\times 10^{3} / \mathrm{mm}^{3}\right)$ & $\mathrm{RBC}\left(\times 10^{6} / \mathrm{mm}^{3}\right)$ & $\mathrm{Hb}(\mathrm{g} / \mathrm{dl})$ \\
\hline Group I & $6.3 \pm 0.25$ & $11.2 \pm 0.38$ & $18.6 \pm 0.43$ \\
Group II & $13.1 \pm 0.53^{\mathrm{a}}$ & $6.8 \pm 0.24^{\mathrm{a}}$ & $10.3 \pm 0.35^{\mathrm{a}}$ \\
Group III & $9.7 \pm 0.33^{\mathrm{b}}$ & $8.5 \pm 0.18^{\mathrm{b}}$ & $14.2 \pm 0.38^{\mathrm{b}}$ \\
Group IV & $7.9 \pm 0.45^{\mathrm{b}, \mathrm{c}}$ & $9.8 \pm 0.26^{\mathrm{b}, \mathrm{c}}$ & $16.8 \pm 0.45^{\mathrm{b}, \mathrm{c}}$ \\
Group V & $7.2 \pm 0.36^{\mathrm{b}, \mathrm{c}}$ & $10.5 \pm 0.52^{\mathrm{b}, \mathrm{c}}$ & $17.8 \pm 0.26^{\mathrm{b}, \mathrm{c}}$ \\
\hline
\end{tabular}

Values expressed as mean \pm standard error of mean $(n=8)$ of hematological changes due to D-carvone administration in CFA-induced rats. Group I = Normal control; Group II = CFA-induced arthritis model; Group III = D-carvone 30 mg/kg b.w. + CFA; Group IV = D-carvone 60 mg/kg b.w. + CFA; Group V = Indomethacin positive control + CFA. CFA, complete Freund's adjuvant; b.w., body weight; WBC, white blood cells; RBC, red blood cells; Hb, hemoglobin. Superscript 'a' indicates significant difference $(p<0.05)$ from Group I, superscript ' $b$ ' indicates significant difference $(p<0.05)$ from Group II, superscript ' $c$ ' indicates significant difference $(p<0.05)$ from Group III, by one-way ANOVA followed by Tukey's multiple comparisons test.

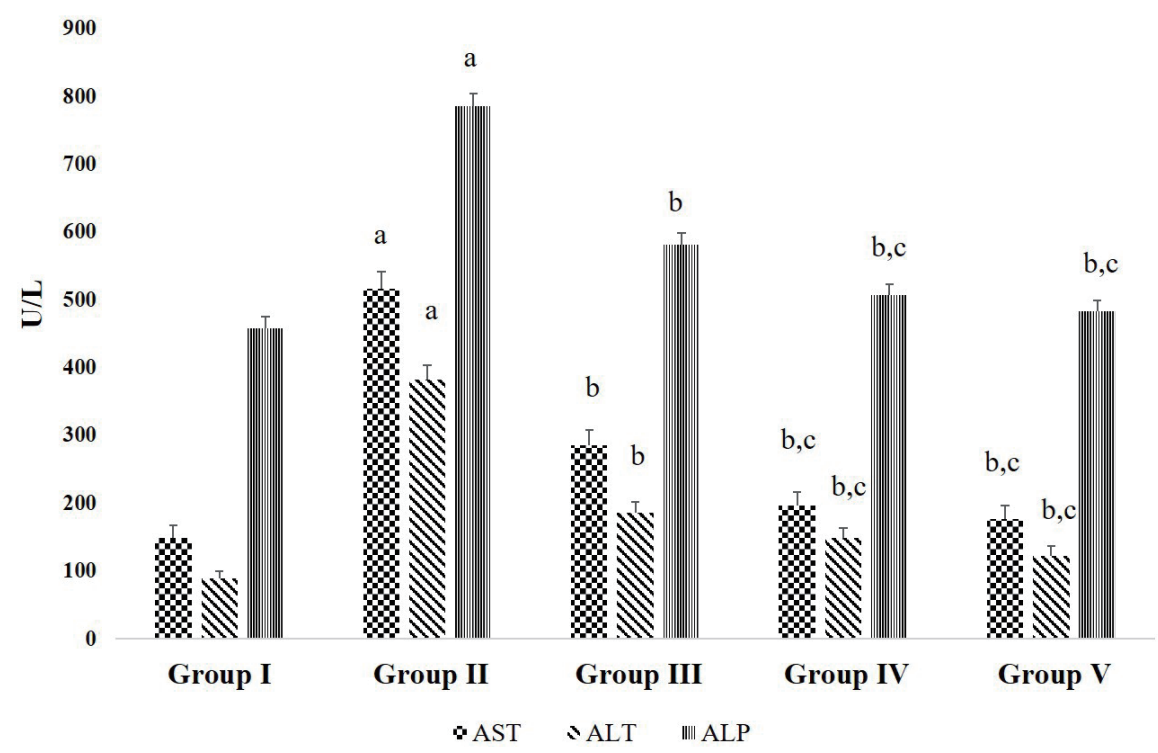

Fig. 3. Effects of $D$-carvone on serum liver markers in CFA-induced arthritic rats. Values expressed as mean \pm standard error of mean ( $n=8$ ) of hematological changes due to D-carvone administration in CFA-induced rats. Group I = Normal control; Group II = CFA-induced arthritis model; Group III = D-carvone 30 mg/kg b.w. + CFA; Group IV = D-carvone 60 mg/kg b.w. + CFA; Group V = Indomethacin positive control + CFA. CFA, complete Freund's adjuvant; b.w., body weight; ALP, alkaline phosphatase; AST, aspartate aminotransferase; ALT, alanine aminotransferase. Superscript 'a' indicates significant difference $(p<0.05)$ from Group I, superscript ' $b$ ' indicates significant difference $(p<0.05)$ from Group II, superscript ' $c$ ' indicates significant difference $(p<0.05)$ from Group III, by one-way ANOVA followed by Tukey's multiple comparisons test.

0.05) the levels of MDA formations in rats of group III and IV compared to arthritic model rats of group II. Levels of and nonenzymatic and enzymatic antioxidants GSH, SOD, and CAT were reduced $(\mathrm{p}<0.05)$ in arthritis model rats of group II in comparison with normal control rats of group I as shown in Fig. 4. The levels of GSH, SOD, and CAT were significantly elevated ( $\mathrm{p}<$ 0.05 ) in D-carvone treated rats of group III and IV as compared to arthritis model rats of group II. Positive control rats of group $\mathrm{V}$ significantly modulated $(\mathrm{p}<0.05)$ the changes in MDA, GSH, SOD, and CAT levels induced by CFA.

\section{Effects of D-carvone on CFA-induced changes of inflammatory cytokines}

The serum levels of pro-inflammatory cytokines IL-6, IL-1 $\beta$,
TNF- $\alpha$, and inflammatory mediator IL-10 were significantly elevated $(\mathrm{p}<0.05)$ in arthritis model group II rats as compared to the normal control group I rats. The levels of IL-6, IL-1 $\beta$, TNF- $\alpha$, and IL-10 were remarkably suppressed $(\mathrm{p}<0.05)$ in D-carvone administered group III and IV rats in contrast to the arthritis model rats. CFA-induced changes in the levels of inflammatory cytokines were reversed $(\mathrm{p}<0.05)$ in the positive control group rats. The results are given in Fig. 5.

\section{Histopathological modifications by D-carvone in CFA- induced arthritic rats}

The histopathological changes of hind paw of rats induced with CFA and treated with D-carvone are displayed in Fig. 6A. The tissue arrangements for normal control group I rats were healthy 

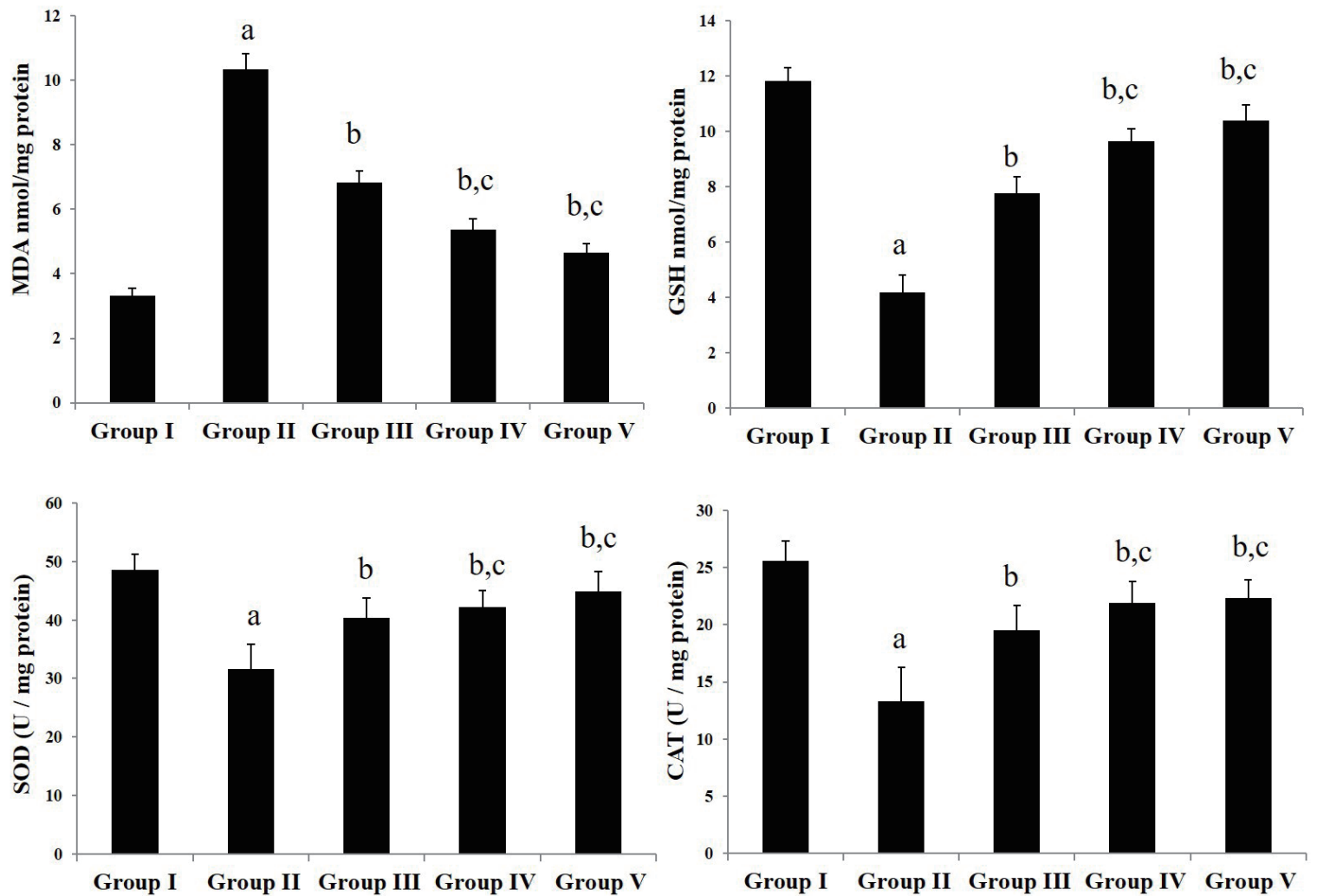

Fig. 4. Effects of $D$-carvone on oxidative stress in CFA-induced arthritic rats. Values expressed as mean \pm standard error of mean ( $n=8$ ) of lipid peroxidation, enzymatic and non-enzymatic antioxidants. Group I = Normal control; Group II = CFA-induced arthritis model; Group III = D-carvone 30 mg/kg b.w. + CFA; Group IV = D-carvone 60 mg/kg b.w. + CFA; Group V = Indomethacin positive control + CFA. CFA, complete Freund's adjuvant; b.w., body weight; MDA, malondialdehyde; GSH, reduced glutathione; SOD, superoxide dismutase; CAT, catalase. Superscript 'a' indicates significant difference $(p<0.05)$ from Group I, superscript ' $b$ ' indicates significant difference $(p<0.05)$ from Group II, superscript ' $c$ ' indicates significant difference $(p<$ $0.05)$ from Group III, by one-way ANOVA followed by Tukey's multiple comparisons test.

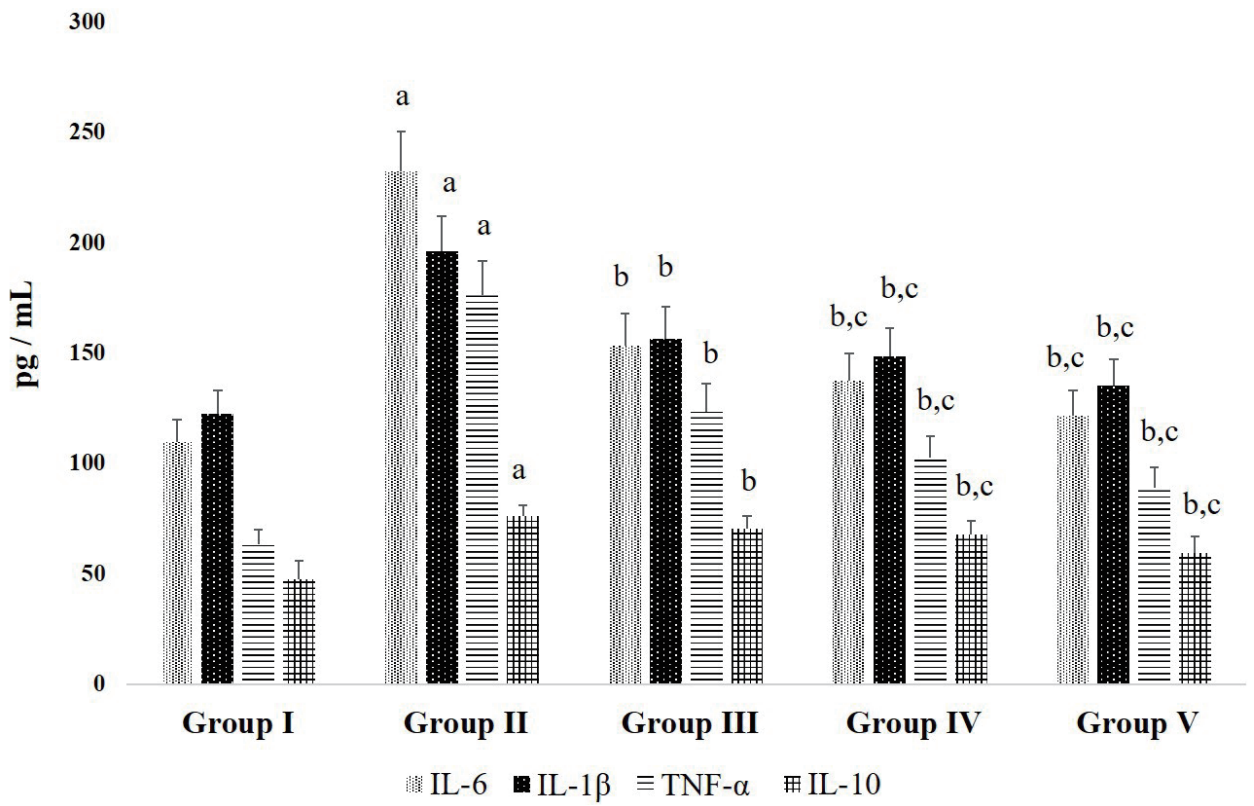

Fig. 5. Effects D-carvone on inflammatory cytokines in CFA-induced arthritic rats. Values expressed as mean \pm standard error of mean ( $n=8$ ) of serum inflammatory cytokines. Group I = Normal control; Group II = CFA-induced arthritis model; Group III = D-carvone 30 mg/kg b.w. + CFA; Group IV = D-carvone 60 mg/kg b.w. + CFA; Group V = Indomethacin positive control + CFA. CFA, complete Freund's adjuvant; b.w., body weight; IL, interleukin; TNF, tumor necrosis factor. Superscript 'a' indicates significant difference $(p<0.05)$ from Group I, superscript ' $b$ ' indicates significant difference $(p<0.05)$ from Group II, superscript ' $c$ ' indicates significant difference $(p<0.05)$ from Group III, by one-way ANOVA followed by Tukey's multiple comparisons test. 


\section{A Normal control}

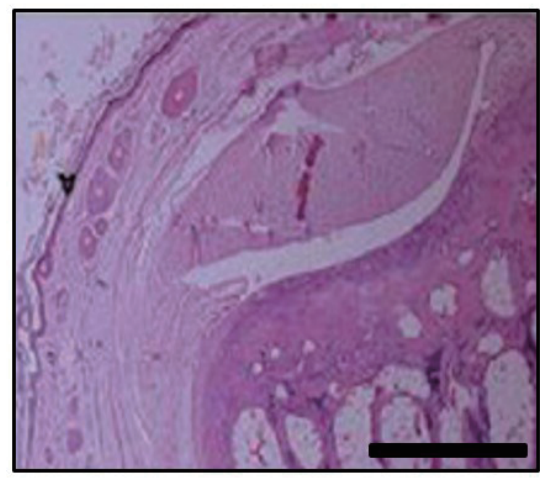

D-carvone $60 \mathrm{mg} / \mathrm{kg}+\mathrm{CFA}$

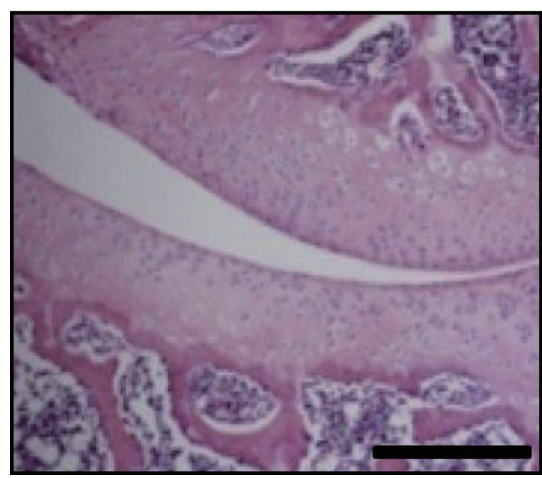

Arthritis model

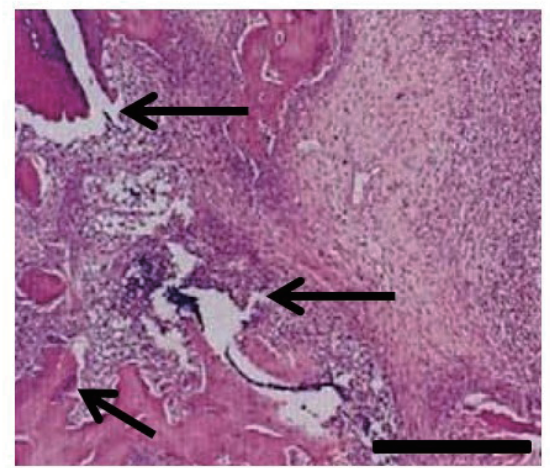

D-carvone $30 \mathrm{mg} / \mathrm{kg}+$ CFA

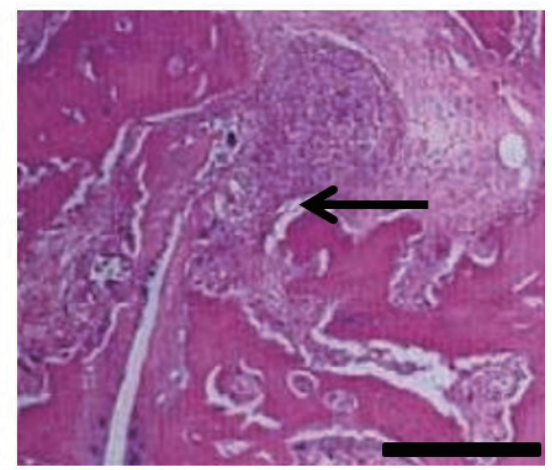

\section{Indomethacin + CFA}

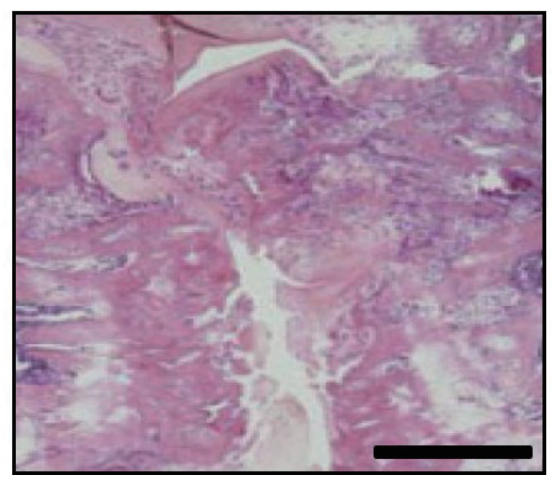

\section{B}

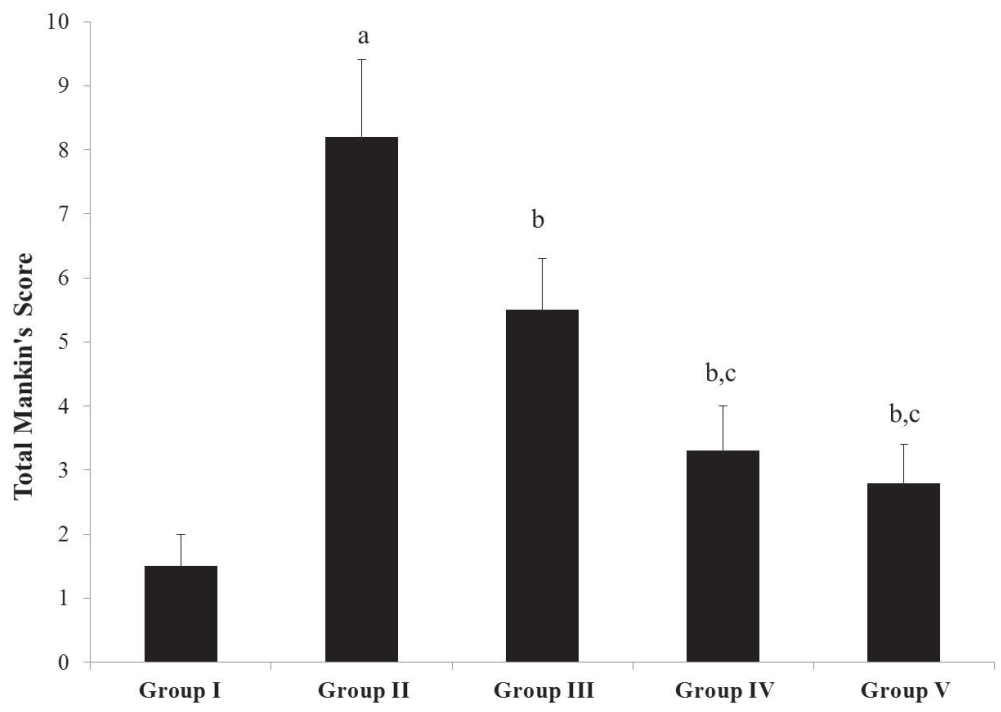

Fig. 6. Modulating effect of D-carvone on the hind paw histopathology of CFA-induced rats. (A) Hind paw sections of arthritis model group II shows severe signs of articular cartilage damage, infiltration of inflammatory cells, synovial hyperplasia, and bone marrow degeneration. Administration of D-carvone in rats of group III and IV ameliorated the CFA-induced arthritic inflammation and changes. Positive control indomethacin group V also exhibited positive changes against CFA-induced arthritic damage. Arrow indicates articular cartilage erosion. Scale bar $=500 \mu \mathrm{m} ; \mathrm{H} \& \mathrm{E}$ staining at 100x magnification. (B) Total Mankin's score for articular injuries and cellular irregularities. Group I = Normal control; Group II = CFA-induced arthritis model; Group III = D-carvone 30 mg/kg b.w. + CFA; Group IV = D-carvone 60 mg/kg b.w. + CFA; Group V = Indomethacin positive control + CFA. CFA, complete Freund's adjuvant; b.w., body weight. Superscript 'a' indicates significant difference $(p<0.05)$ from Group l, superscript ' $b$ ' indicates significant difference $(p<0.05)$ from Group II, superscript ' $c$ ' indicates significant difference $(p<0.05)$ from Group III, by one-way ANOVA followed by Tukey's multiple comparisons test. 
with distinct synovial arrangements and joint spaces. In contrast, the arthritis model group II rats exhibited severe inflammation with edema, articular cartilage damage, synovial hyperplasia, infiltration of inflammatory cells, and bone marrow degeneration. The pathology of hind paw of rats treated with D-carvone in group III and IV showed marked reduction in the inflammatory cells infiltration, preserved articular cartilage, regular bone marrow, and reduced synovial hyperplasia. Positive control rats of group $\mathrm{V}$ also exhibited reversal in the pathological changes of hind paw caused by CFA-induced arthritis. Arrows in the histopathology of CFA-induced arthritic rats show articular cartilage erosions. The total Mankin's scoring for articular injuries and cellular irregularities is given in Fig. 6B. Mankin's scores were relatively high for group II compared to group I, with relevance to the morphological changes observed. D-carvone significantly lowered the Mankin's scores dose-dependently, compared to group II. Mankin's scores for group V were also lowered in agreement with the morphological observations.

\section{DISCUSSION}

Medical treatment for osteoarthritis depends on non-steroidal anti-inflammatory drugs (NSAIDs) for pain-relief and prevention of inflammation. Prolonged treatment with NSAIDs leads to unwanted side effects on renal, cardiovascular, gastrointestinal, and other major organs [16]. Due to the adverse effects of NSAIDs and re-occurrence of osteoarthritis due to prolonged cartilage degeneration, there is need for an alternative treatment method. Therefore, antioxidants which are natural anti-inflammatory agents with least or no side effects are being studied to treat osteoarthritic conditions [17]. D-carvone was proven with multiple therapeutic potentials including anti-inflammatory effects $[11,12]$. The results from this research showed D-carvone was able to protect against the osteoarthritis induced by CFA in rats. Paw volume and arthritic index of CFA-induced rats were significantly reduced by $\mathrm{D}$-carvone in a dose-dependent manner proving the capability of the antioxidant compound in preventing inflammation. CFA is known to induce chronic inflammation by upregulating the migration of lymphocytes and macrophages along with the release of inflammatory mediators such as TNF- $\alpha$, IL- 6 , IL-1 $\beta$, and IL-10 $[15,18]$. These mediators in turn, cause damage to the tissue and articular cartilages, leading to osteoarthritic conditions [19]. These changes were observed in the results of the CFA-induced model rats, with inflammatory cell infiltration in histopathological results and elevation of inflammatory mediators in ELISA analysis. Administration of D-carvone reduced the infiltration of inflammatory cells as well suppressed the inflammatory mediators suggesting that $\mathrm{D}$-carvone was able to reverse the osteoarthritic condition induced by CFA. The histopathological results also prove that D-carvone was able to prevent the articular cartilage damage and synovial hyperplasia induced by
CFA. Many researchers suggest that the release of inflammatory mediators could trigger the immune response during osteoarthritic inflammation [20,21]. Thymus and spleen are important organs for immune response and regulation, which acts to produce immune cells and filters the dead cells [15]. Increase in the organ index of spleen and thymus in CFA-induced model rats could be explained with the filtration of immune cells along with dead RBCs and WBCs. The results show that levels of blood cells were altered by CFA-induced inflammation. WBCs increase in count by a stimulation during elevation of inflammatory cytokines especially IL-1 $\beta$ [20]. Arthritic conditions are commonly related to anemia due to deformation of RBCs. Hemoglobin levels decrease during osteoarthritic conditions due to deformation of RBCs and diminished erythropoietin levels due to bone marrow degeneration [22]. These features were prominent in the CFAinduced model rats as displayed by the histopathological results and hematological changes. D-carvone remarkably attenuated the hematological changes caused by CFA by preventing bone marrow degeneration and reduced the expressions of inflammatory mediator. The thymus and spleen organ index were also reduced in D-carvone treated rats as a result of reduced dead cells suppressed immune response.

Oxidative stress is considered as a progenitor in osteoarthritis. The prevention of oxidative stress has been reported to prevent the osteoarthritic conditions in animal models $[5,6,9]$. From the results, it was clearly observed that the level of lipid peroxidation was elevated whereas the levels of enzymatic and non-enzymatic antioxidants were reduced in the CFA-induced model rats indicating that the rats were under oxidative stress. Researchers have suggested that inflammatory cells such as granulocytes and macrophages tend to manifest synovial fluid in the inflamed area of CFA-induced osteoarthritis, which produces oxygen and hydrogen peroxide free radicals [23]. Inflammation of ankle joints in osteoarthritic conditions is directly influenced by the lipid peroxidation activity of free radicals. Oxidative stress triggers the release of inflammatory mediators by initiating the NF-k $\beta$ pathway activation. Triggering of inflammatory responses leads to tissue and articular cartilage damage in osteoarthritic conditions [24]. D-carvone as an antioxidant was able to prevent oxidative stress by revitalizing the endogenous antioxidants and preventing lipid peroxidation. CFA-induced osteoarthritic model rats also exhibited liver damage as evidenced by the serum biochemical results with increased AST, ALT, and ALP levels which can also be attributed to the oxidative stress condition. The therapeutic dose of $\mathrm{D}$-carvone oral administration in modulation of skin tumorigenesis and colon cancer in rat models was at $20 \mathrm{mg} / \mathrm{kg}$ and $10 \mathrm{mg} / \mathrm{kg}$ respectively [11,12]. A recent study on ameliorative effect of oral administration of D-carvone on lung inflammation in mice model was proven effective at the dose of $50 \mathrm{mg} / \mathrm{kg}$ [25]. In this study, the effective dose of D-carvone oral administration to protect against CFA-induced arthritis is at $60 \mathrm{mg} / \mathrm{kg}$. Hence, in order to achieve required therapeutic effect, the oral dosage of 
D-carvone needs to be optimized. Monoterpenes are reported to have high bioavailability upon oral administration in rats [26,27]. Being a monoterpene, D-carvone is also presumed to have high bioavailability after oral administration. Remarkably, administration of D-carvone was able to reverse the CFA-induced serum biochemical changes, indicating D-carvone might have protected liver from oxidative stress.

Collectively, the results obtained from this study demonstrate the anti-arthritic activity of D-carvone against CFA-induced arthritis in rats. Prevention of oxidative stress, inflammatory cell infiltration, and pathological changes by $\mathrm{D}$-carvone have suppressed the release of inflammatory cytokines and inflammatory response thus avoiding damage to the tissues and articular cartilages. D-carvone could serve as a therapeutic drug for inflammatory and arthritic conditions. Detailed antioxidative mechanism of D-carvone on arthritic conditions needs to be elucidated in future research.

\section{CONFLICTS OF INTEREST}

The authors declare no conflicts of interest.

\section{REFERENCES}

1. Poulet B, Staines KA. New developments in osteoarthritis and cartilage biology. Curr Opin Pharmacol. 2016;28:8-13.

2. Blasioli DJ, Kaplan DL. The roles of catabolic factors in the development of osteoarthritis. Tissue Eng Part B Rev. 2014;20:355-363.

3. Wojdasiewicz P, Poniatowski ŁA, Szukiewicz D. The role of inflammatory and anti-inflammatory cytokines in the pathogenesis of osteoarthritis. Mediators Inflamm. 2014;2014:561459.

4. Wang S, Wang Y, Liu X, Guan L, Yu L, Zhang X. Anti-inflammatory and anti-arthritic effects of taraxasterol on adjuvant-induced arthritis in rats. J Ethnopharmacol. 2016;187:42-48.

5. Jeong JH, Moon SJ, Jhun JY, Yang EJ, Cho ML, Min JK. Eupatilin exerts antinociceptive and chondroprotective properties in a rat model of osteoarthritis by downregulating oxidative damage and catabolic activity in chondrocytes. PLoS One. 2015;10:e130882.

6. Park JS, Kim DK, Shin HD, Lee HJ, Jo HS, Jeong JH, Choi YL, Lee CJ, Hwang SC. Apigenin regulates interleukin-1 $\beta$-induced production of matrix metalloproteinase both in the knee joint of rat and in primary cultured articular chondrocytes. Biomol Ther (Seoul). 2016;24:163-170.

7. Wang SX, Abramson SB, Attur M, Karsdal MA, Preston RA, Lozada CJ, Kosloski MP, Hong F, Jiang P, Saltarelli MJ, Hendrickson BA, Medema JK. Safety, tolerability, and pharmacodynamics of an anti-interleukin- $1 \alpha / \beta$ dual variable domain immunoglobulin in patients with osteoarthritis of the knee: a randomized phase 1 study. Osteoarthritis Cartilage. 2017;25:1952-1961.

8. Wu Y, Goh EL, Wang D, Ma S. Novel treatments for osteoarthritis: an update. Open Access Rheumatol. 2018;10:135-140.

9. Huang X, Xi Y, Pan Q, Mao Z, Zhang R, Ma X, You H. Caffeic acid protects against IL-1 $\beta$-induced inflammatory responses and cartilage degradation in articular chondrocytes. Biomed Pharmacother. 2018;107:433-439.

10. Lin B, Zhao Y, Han P, Yue W, Ma XQ, Rahman K, Zheng CJ, Qin LP, Han T. Anti-arthritic activity of Xanthium strumarium L. extract on complete Freund's adjuvant induced arthritis in rats. J Ethnopharmacol. 2014;155:248-255.

11. Gopalakrishnan T, Ganapathy S, Veeran V, Namasivayam N. Preventive effect of $\mathrm{D}$-carvone during DMBA induced mouse skin tumorigenesis by modulating xenobiotic metabolism and induction of apoptotic events. Biomed Pharmacother. 2019;111:178-187.

12. Vinothkumar R, Sudha M, Viswanathan P, Kabalimoorthy J, Balasubramanian T, Nalini N. Modulating effect of D-carvone on 1,2-dimethylhydrazine-induced pre-neoplastic lesions, oxidative stress and biotransforming enzymes, in an experimental model of rat colon carcinogenesis. Cell Prolif. 2013;46:705-720.

13. Moro IJ, Gondo GD, Pierri EG, Pietro RC, Soares CP, Sousa DP, dos Santos AG. Evaluation of antimicrobial, cytotoxic and chemopreventive activities of carvone and its derivatives. Braz J Pharm Sci. 2018;53:e00076.

14. de Cássia da Silveira e Sá R, Andrade LN, de Sousa DP. A review on anti-inflammatory activity of monoterpenes. Molecules. 2013;18: 1227-1254.

15. Chen Y, Xue R, Jin X, Tan X. Antiarthritic activity of diallyl disulfide against Freund's adjuvant-induced arthritic rat model. J Environ Pathol Toxicol Oncol. 2018;37:291-303.

16. Rahmati M, Mobasheri A, Mozafari M. Inflammatory mediators in osteoarthritis: a critical review of the state-of-the-art, current prospects, and future challenges. Bone. 2016;85:81-90.

17. Patil MVK, Kandhare AD, Bhise SD. Anti-arthritic and anti-inflammatory activity of Xanthium srtumarium L. ethanolic extract in Freund's complete adjuvant induced arthritis. Biomed Aging Pathol. 2012;2:6-15.

18. Cui X, Wang R, Bian P, Wu Q, Seshadri VDD, Liu L. Evaluation of antiarthritic activity of nimbolide against Freund's adjuvant induced arthritis in rats. Artif Cells Nanomed Biotechnol. 2019;47:3391-3398.

19. Zhang X, Dong Y, Dong H, Zhang W, Li F. Investigation of the effect of phlomisoside F on complete Freund's adjuvant-induced arthritis. Exp Ther Med. 2017;13:710-716.

20. Lima-Garcia JF, Dutra RC, da Silva K, Motta EM, Campos MM, Calixto JB. The precursor of resolvin D series and aspirin-triggered resolvin D1 display anti-hyperalgesic properties in adjuvant-induced arthritis in rats. BrJ Pharmacol. 2011;164:278-293.

21. Zhang L, Zhu M, Li M, Du Y, Duan S, Huang Y, Lu Y, Zhang J, Wang T, Fu F. Ginsenoside Rg1 attenuates adjuvant-induced arthritis in rats via modulation of PPAR- $\gamma / \mathrm{NF}-\kappa \mathrm{B}$ signal pathway. Oncotarget. 2017;8:55384-55393.

22. Qiao YQ, Jiang PF, Gao YZ. Lutein prevents osteoarthritis through Nrf2 activation and downregulation of inflammation. Arch Med Sci. 2018;14:617-624.

23. Hou SM, Hou CH, Liu JF. CX3CL1 promotes MMP-3 production via the CX3CR1, c-Raf, MEK, ERK, and NF- $\mathrm{KB}$ signaling pathway in osteoarthritis synovial fibroblasts. Arthritis Res Ther. 2017;19:282.

24. Jiang L, Li L, Geng C, Gong D, Jiang L, Ishikawa N, Kajima K, Zhong L. Monosodium iodoacetate induces apoptosis via the mitochondrial pathway involving ROS production and caspase activa- 
tion in rat chondrocytes in vitro. J Orthop Res. 2013;31:364-369.

25. Zhao M, Du J. Anti-inflammatory and protective effects of Dcarvone on lipopolysaccharide (LPS)-induced acute lung injury in mice. J King Saud Univ Sci. 2020;32:1592-1596.

26. Marchese A, Arciola CR, Barbieri R, Silva AS, Nabavi SF, Tsetegho Sokeng AJ, Izadi M, Jafari NJ, Suntar I, Daglia M, Nabavi SM. Update on monoterpenes as antimicrobial agents: a particular focus on p-cymene. Materials (Basel). 2017;10:947.

27. Pavan B, Dalpiaz A, Marani L, Beggiato S, Ferraro L, Canistro D, Paolini M, Vivarelli F, Valerii MC, Comparone A, De Fazio L, Spisni E. Geraniol pharmacokinetics, bioavailability and its multiple effects on the liver antioxidant and xenobiotic-metabolizing enzymes. Front Pharmacol. 2018;9:18. 